\title{
EVALUATION OF 30 CASES OF BRONCHIECTASIS USING HRCT
}

\author{
Japnit Singh ${ }^{1}$, Ashutosh Chitnis², Rajiv Hira ${ }^{3}$ \\ ${ }^{1}$ Third Year Resident, Department of Radiology, MGM Medical College, Navi Mumbai. \\ 2 Professor, Department of Radiology, MGM Medical College, Navi Mumbai. \\ ${ }^{3}$ Senior Resident, Department of Radiology, MGM Medical College, Navi Mumbai.
}

\begin{tabular}{l}
\hline ABSTRACT \\
BACKGROUND \\
The objective of this study is to identify the most common aetiology of bronchiectasis in patients undergoing HRCT chest at Navi \\
Mumbai. Further, to understand the demographical profile and anatomical distribution of bronchiectasis in these patients.
\end{tabular}

\section{MATERIALS AND METHODS}

This study included 30 patients within the age group of 10 - 75 years who had all undergone an HRCT chest for a variety of chest symptoms such as breathlessness, cough, haemoptysis and fever with/without chills. These patients were scanned using a 64 slice Toshiba Aquilion CT machine. The scans were done in the supine position. For inspiratory scans, $1 \mathrm{~mm}$ thick sections were taken at $5 \mathrm{~mm}$ intervals from the lung apices to below the CP angles with $50 \mathrm{mAs}, 120 \mathrm{kVp}$. Same parameters were used for the expiratory scans except in this the interval taken was $20 \mathrm{~mm}$.

\section{RESULTS}

In this study, the male: female ratio was found to be 3: 2 . The cause of bronchiectasis in most patients in this study was found to be post-infectious, Koch's being the commonest (66.67\%) followed by Staphylococcus infection (13.33\%), UIP (10\%), fungal infection $(6.67 \%)$ and COP (3.33\%). The left lung was more commonly involved (56.67\%) followed by the right lung (26.67\%) and then bilateral involvement (16.67\%). The upper lobes were most commonly involved (43.3\%) followed by the lower lobes $(36.6 \%)$. Middle lobe involvement was 20\%. Other associated findings included tree in bud appearance in 10 cases, air space consolidation in 6 cases, mucous plugs in 3 cases, pleural effusion and cavity in 2 cases each.

\section{CONCLUSION}

It is important to perform HRCT in symptomatic cases of suspected bronchiectasis. HRCT is a very sensitive modality for showing bronchiectasis which is characterised by the lack of bronchial tapering, bronchi visible in the peripheral $1 \mathrm{~cm}$ of the lungs and an increased broncho-arterial ratio producing the 'signet-ring sign.' Bronchiectasis is caused by a complex cycle of repeated inflammation and a defective immune response to suppress it. Haemoptysis is common and is usually a sequelae of tuberculosis. Commonly bronchiectasis results from necrotising bacterial infections, but in this study Koch's infection evolved as the most common cause of bronchiectasis.

\section{KEYWORDS}

HRCT, Bronchiectasis, Tuberculosis, Koch's, Infection.

HOW TO CITE THIS ARTICLE: Singh J, Chitnis A, Hira R. Evaluation of 30 cases of bronchiectasis using HRCT. J. Evolution Med. Dent. Sci. 2017;6(13):1052-1055, DOI: 10.14260/Jemds/2017/226

\section{BACKGROUND}

Bronchiectasis is the irreversible, abnormal dilatation of the bronchi.

Involved bronchi are dilated, inflamed and easily collapsible, resulting in airflow obstruction and impaired clearance of secretions.

\section{Aims and Objectives}

1. To identify the most common aetiology of bronchiectasis in patients undergoing HRCT chest at Navi Mumbai.

2. To understand the demographical profile and anatomical distribution of bronchiectasis in these patients.

Financial or Other, Competing Interest: None.

Submission 25-10-2016, Peer Review 15-01-2017,

Acceptance 21-01-2017, Published 13-02-2017.

Corresponding Author:

Dr. Japnit Singh,

PG Hostel, MGM Medical College,

Kamothe,

Navi Mumbai-410209.

E-mail: japosingh@gmail.com

DOI: $10.14260 /$ jemds $/ 2017 / 226$

\section{Causes}

- Post infectious (most common).

- Congenital.

- Bronchial obstruction.

- Others.

High resolution CT is a scanning protocol, in which thin sections (usually 0.625 to $1.25 \mathrm{~mm}$ ) are acquired and reconstructed using a sharp algorithm (e.g. bone algorithm).

\section{It has been used for}

- Lung imaging.

- Temporal bone imaging.

\section{MATERIALS AND METHODS}

This study included 30 patients within the age group of 10 75 years, who had all undergone an HRCT chest.

\section{They all had a Variety of Chest Symptoms}

- Breathlessness.

- Cough with variable amount expectoration (scanty to copious).

- Haemoptysis. 
- Fever with/without chills.

\section{Equipment and Protocol}

- These patients were scanned using a 64 slice Toshiba Aquilion CT machine.

- The scans were done in the supine position.

- For inspiratory scans, $1 \mathrm{~mm}$ thick sections were taken at $5 \mathrm{~mm}$ intervals from the lung apices to below the CP angles with $50 \mathrm{mAs}, 120 \mathrm{kVp}$.

- Same parameters were used for the expiratory scans, except in this the interval taken was $20 \mathrm{~mm}$.

\section{RESULTS}

\begin{tabular}{|c|c|c|}
\hline $\begin{array}{c}\text { HRCT } \\
\text { Findings }\end{array}$ & $\begin{array}{c}\text { No. of } \\
\text { Cases }\end{array}$ & $\begin{array}{c}\text { Percentage of } \\
\text { Cases }\end{array}$ \\
\hline Bronchiectasis & 30 & $100 \%$ \\
\hline Consolidation & 6 & $20 \%$ \\
\hline Tree in Bud & 10 & $33 \%$ \\
\hline Pleural Effusion & 2 & $6.7 \%$ \\
\hline Cavity & 2 & $6.7 \%$ \\
\hline Secretions & 3 & $10 \%$ \\
\hline \multicolumn{2}{|c|}{ Table 1. Summary of HRCT Findings }
\end{tabular}

\begin{tabular}{|c|c|c|c|}
\hline Disease & $\begin{array}{c}\text { Total No. } \\
\text { of Cases }\end{array}$ & $\begin{array}{c}\text { No. of } \\
\text { Male Patients }\end{array}$ & $\begin{array}{c}\text { No. of } \\
\text { Female Patients }\end{array}$ \\
\hline Tuberculosis & 20 & 13 & 7 \\
\hline UIP & 3 & 2 & 1 \\
\hline $\begin{array}{c}\text { Staph. } \\
\text { Infection }\end{array}$ & 4 & 2 & 2 \\
\hline $\begin{array}{c}\text { Fungal } \\
\text { Infection }\end{array}$ & 2 & 1 & 1 \\
\hline COP & 1 & 0 & 1 \\
\hline \multicolumn{2}{|c|}{ Table 2. Demographical Distribution based on Aetiology } \\
\hline
\end{tabular}

\begin{tabular}{|c|c|}
\hline $\begin{array}{c}\text { Distribution of Lesions } \\
\text { in Bronchiectasis }\end{array}$ & $\begin{array}{c}\text { No. of } \\
\text { Cases }\end{array}$ \\
\hline Right Lung & 8 \\
\hline Left Lung & 17 \\
\hline Both Lungs & 5 \\
\hline Upper Lobes & 13 \\
\hline Lower Lobes & 11 \\
\hline Middle Lobe & 6 \\
\hline \multicolumn{2}{|c|}{ Table 3. Distribution of Lesions } \\
\hline
\end{tabular}

\section{DISCUSSION}

High resolution CT is a scanning protocol in which thin sections (usually 0.625 to $1.25 \mathrm{~mm}$ ) are acquired and reconstructed using a sharp algorithm (e.g. bone algorithm). It has been used for:

- Lung imaging.

- Temporal bone imaging.

\section{Lung Imaging}

Two techniques have been used.

- $\quad$ Spaced axial (Non-helical).

- Volumetric HRCT.

\section{Spaced Axial}

Thin sections are acquired with an interval of $1-2 \mathrm{cms}$ between the two sets of images. It has been considered sufficient to detect abnormalities in diffuse lung diseases. ${ }^{1}$ This technique is most useful when single detector CT is being used. The patient dose is less as compared to volumetric imaging.

\section{Volumetric HRCT}

Thin sections are acquired continuously using multidetector row CT scanners and in a single breath-hold. It allows detection of all the abnormalities and has been considered easier and better for interpretation by providing the ability for coronal and sagittal reformations. The patient dose though is higher as compared to axial imaging.

\section{Fundamental Technical Protocols}

Slice Thickness $-0.625-1.25 \mathrm{~mm}$

Scan Time - 0.5 - 1 second

$\mathbf{k V}-120$

mAs - 100 - 200

Collimation - $1.5-3 \mathrm{~mm}$

Matrix Size -768 x 768 or the largest available

FOV - $35 \mathrm{~cm}$

Reconstruction Algorithm - High spatial frequency

Window - Lung window

Patient Position - Supine (Routinely) or prone (If suspected ILD)

\section{Level of Inspiration}

Full inspiration (Routinely recommended) expiratory HRCT scans at three or more levels in patients with obstructive lung diseases.

\section{Bronchiectasis}

Bronchiectasis refers to abnormal dilatation of the bronchial tree and is seen in a variety of clinical settings. CT is the most accurate modality for diagnosis. It is largely considered irreversible. $^{2}$

As there are many causes of bronchiectasis, which may occur at essentially any age no single demographic is particularly involved. Two groups make up the majority of cases: post-infectious and cystic fibrosis.

Bronchiectasis typically presents with recurrent chest infections, production of copious amounts of sputum and haemoptysis. The latter is often the only symptom and is encountered in up to $50 \%$ of cases.

As is true for most lung pathology, bronchiectasis is the common response of bronchi to many different insults. Causes include -

\section{Post-Infective (Most Common)}

- Necrotising bacterial pneumonia, e.g. Staph. aureus, Klebsiella, B. pertussis.

- Granulomatous disease, e.g. tuberculosis, MAIC, histoplasmosis.

- $\quad$ Allergic Bronchopulmonary Aspergillosis (ABPA).

- Measles.

\section{Congenital}

- Congenital cystic bronchiectasis.

- Cystic Fibrosis (CF).

- Ciliary dysfunction syndromes, e.g. Kartagener syndrome.

- Bronchial cartilage deficiency, e.g. Williams-Campbell syndrome.

- Abnormal lung, e.g. pulmonary sequestration.

- Abnormal physiology, e.g. alpha-1-antitrypsin deficiency.

- Mounier-Kuhn syndrome. 


\section{Bronchial Obstruction}

- Malignancy, e.g. bronchogenic carcinoma.

- Inhaled foreign bodies.

- Chronic aspiration lung changes.

\section{Loss of Surrounding Lung Volume}

- Traction bronchiectasis.

Others

- $\quad$ Rheumatoid arthritis 6.

- $\quad$ Systemic Lupus Erythematosus (SLE) 7.

- Post-radiotherapy.

- Post-transplantation.

\section{Subtypes}

According to macroscopic morphology, three types have been described, which also represent a spectrum of severity.

\section{Cylindrical Bronchiectasis}

Bronchi have a uniform calibre, do not taper and have parallel walls (Tram track sign and signet ring sign). It is the commonest form.

\section{Varicose Bronchiectasis}

Relatively uncommon, beaded appearances where dilated bronchi have interspersed sites of relative narrowing.

\section{Cystic Bronchiectasis}

Severe form with cyst-like bronchi that extend to the pleural surface air-fluid levels are commonly present.

Additionally, bronchiectasis can result from the traction of fibrotic lung surrounding an airway. ${ }^{3}$ This is known as traction bronchiectasis. There is dilation of bronchi and bronchioles due to elastic tissue and smooth muscle destruction.

CT and HRCT, especially excels at demonstrating the airways and is able to a greater or lesser degree to distinguish some of the various underlying causes.

\section{Several Features are Helpful in Diagnosing} Bronchiectasis ${ }^{4,5}$

Bronchus Visualised within $1 \mathrm{~cm}$ of Pleural Surface -

- Especially true of lung adjacent to costal pleura.

- Most helpful sign for early cylindrical change.

\section{Lack of Tapering}

\section{Increased Bronchoarterial Ratio}

- Diameter of a bronchus should measure approximately 0.65 - 1.0 times that of the adjacent pulmonary artery branch.

- Between 1 and 1.5 may be seen in normal individuals, especially those living at high altitude.

- Greater than 1.5 indicates bronchiectasis.

\section{Several Ancillary Findings are also Recognised}

- Bronchial wall thickening: normally wall of bronchus should be less than half the width of the accompanying pulmonary artery branch.

- Mucoid impaction.

- Air-trapping and mosaic perfusion.
- $\quad$ Signet ring sign.

- String of pearls sign.

- Cluster of grapes sign.

In general treatment of bronchiectasis is medical and involves promoting sputum clearance using positional physiotherapy and early and aggressive treatment of pulmonary infections. ${ }^{6,7}$ In some cases, chronic prophylactic administration of antibiotics may be required.

In cases where bronchiectasis is severe and significant morbidity is present, surgical resection of the affected lobe may be of benefit provided adequate respiratory reserve exists.

In cases where both lungs are extensively involved (e.g. cystic fibrosis) lung transplantation can be considered.

Conditions that may mimic cystic bronchiectasis include:

- Langerhans Cell Histiocytosis (LCH).

- Lymphangiomyomatosis (LAM).

- $\quad$ Lymphocytic Interstitial Pneumonitis (LIP).

- Pulmonary manifestations of AIDS.

- Pneumocystis pneumonia (PCP).

- Emphysema.

- Cystic pulmonary metastases.

\section{Case Findings Examples}

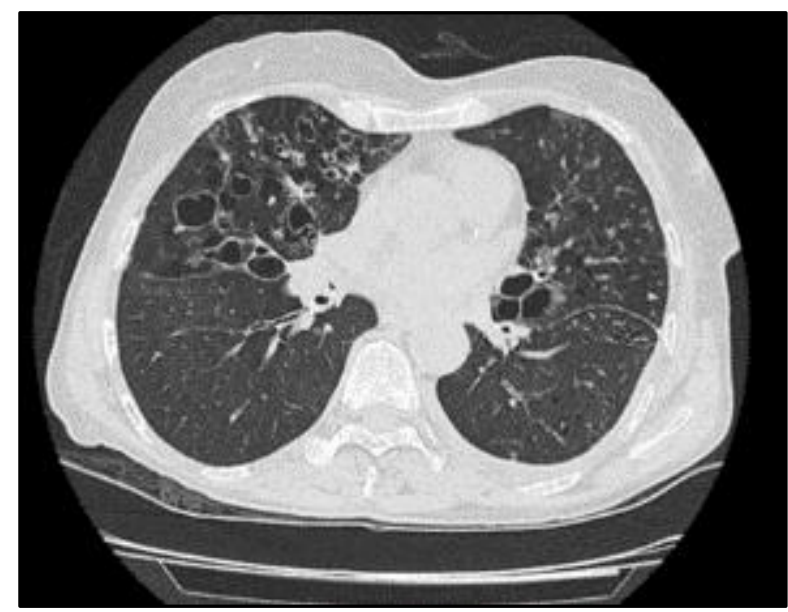

Figure 1. Cystic Bronchiectatic Cavities noted in ML and Post Seg OG LUL

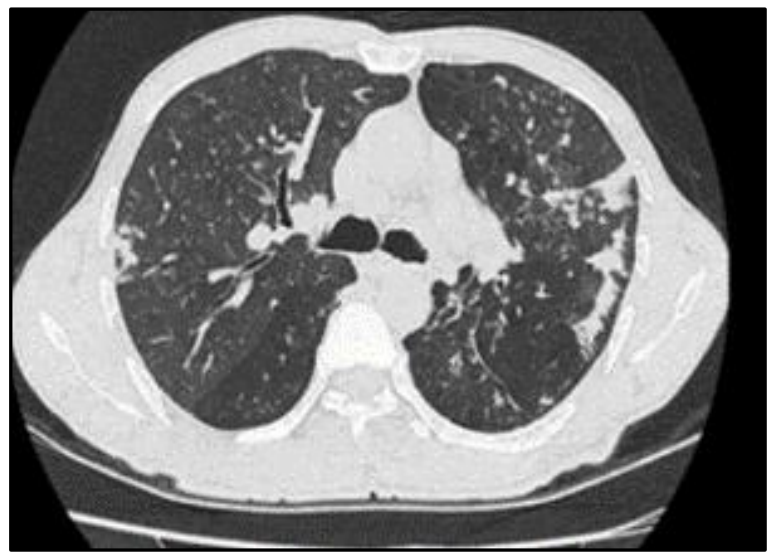

Figure 2. Tree in Bud Nodules, Patchy ASC noted in Post Seg of b/I UL ( $L>R)$

Signs Described on CT include

- Tram-track sign. 


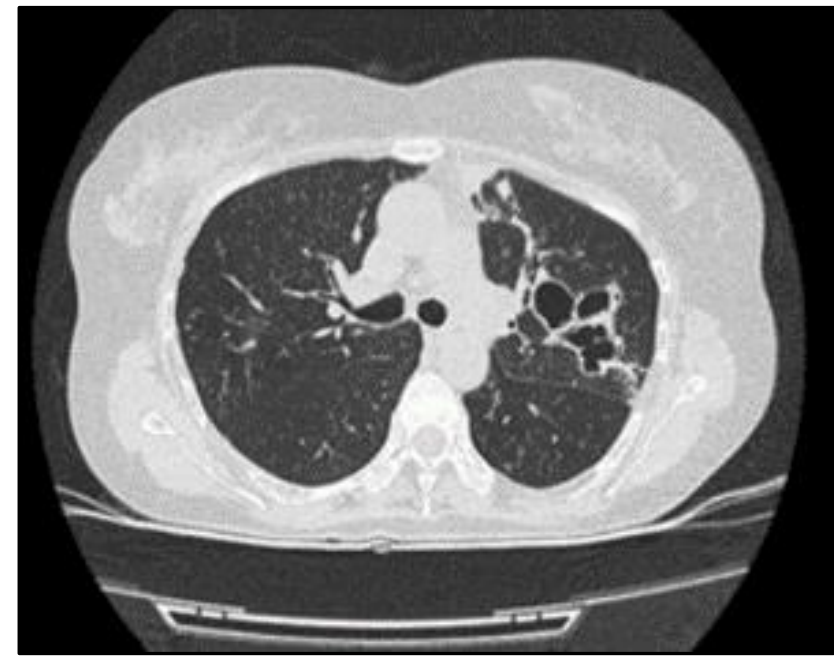

Figure 3. Cystic and Varicose Bronchiectasis in Post Seg of LUL

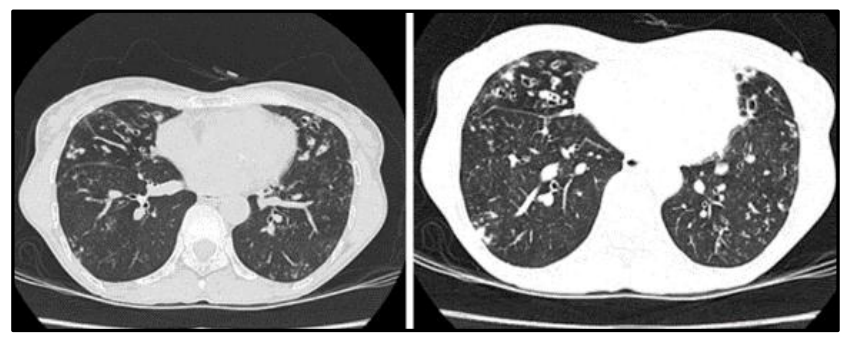

Figure 4. Cystic Bronchiectasis with Signet Ring Sign seen in Medical Seg of ML and Inferior Seg of Lingular Lobe

\section{CONCLUSION}

It is important to perform HRCT in symptomatic cases of suspected bronchiectasis.

High resolution CT is a very sensitive modality for showing bronchiectasis.

It is characterised by the lack of bronchial tapering, bronchi visible in the peripheral $1 \mathrm{~cm}$ of the lungs, and an increased broncho-arterial ratio producing the 'signet-ring sign.'

According to appearance, bronchiectasis can be classified as cylindrical, varicose or cystic.

Bronchiectasis is caused by a complex cycle of repeated inflammation and a defective immune response to suppress it.
Haemoptysis is common and may occur in as many as $50 \%$ of patients. Episodic haemoptysis with little to no sputum production (dry bronchiectasis) is usually a sequelae of tuberculosis.

Commonly bronchiectasis results from necrotising bacterial infections, such as caused by the Staphylococcus or Klebsiella species.

However, in our study, Koch's infection evolved as the most common cause of bronchiectasis. In this study, the male: female ratio was found to be $3: 2$.

The cause of bronchiectasis in most patients in this study was found to be post-infectious, Koch's being the commonest (66.67\%) followed by Staphylococcus infection (13.33\%), UIP (10\%), fungal infection (6.67\%) and COP (3.33\%).

The left lung was more commonly involved (56.67\%) followed by the right lung (26.67\%) and then bilateral involvement (16.67\%).

The upper lobes were most commonly involved (43.3\%) followed by the lower lobes (36.6\%). Middle lobe involvement was $20 \%$.

Other associated findings included tree in bud appearance in 10 cases, air space consolidation in 6 cases, mucous plugs in 3 cases, pleural effusion and cavity in 2 cases each.

\section{REFERENCES}

[1] Kang EY, Miller RR, Müller NL. Bronchiectasis: comparison of preoperative thin-section CT and pathologic findings in resected specimens. Radiology 1995;195(3):649-54.

[2] Reid LM. Reduction in bronchial subdivision in bronchiectasis. Thorax 1950;5(3):233-47.

[3] Westcott JL, Cole SR. Traction bronchiectasis in endstage pulmonary fibrosis. Radiology 1986;161(3):6659.

[4] Hansell DM, Bankier AA, MacMahon H, et al. Fleischner society: glossary of terms for thoracic imaging. Radiology 2008;246(3):697-722.

[5] Barker AF. Bronchiectasis. New Engl J Med 2002;346(18):1383-93.

[6] Baydarian M, Walter RN. Bronchiectasis: introduction, etiology, and clinical features. Dis Mon 2008;54(8):516-26.

[7] Javidan-Nejad C, Bhalla S. Bronchiectasis. Radiol Clin North Am 2009;47(2):289-306. 